\title{
A Near-Death Experience in Pu Songling's Strange Stories from Liaozhai's Studio
}

\author{
Juan S. Gómez-Jeria, Lic. Q. \\ University of Chile
}

ABSTRACT: I present in this paper a tale from Pu Songling's "Strange Stories from Liaozhai's Studio." This story seems to contain the following key elements of a near-death experience: the life review, travel through a spiritual world, and a pilgrimage to obtain a healthy physical body "to return to life." I discuss the content of the life review in terms of emotionally tagged souvenirs. Other contents of the story are clearly culturally dependent and I discuss them within the framework of Oriental thought.

KEY WORDS: near-death experience; Pu Songling; culturally-shaped experience; field of consciousness; long-term memory.

Pu Songling (P'u Sung-ling, 1640-1715), the great Chinese writer, was born in Zibo and lived in Pujia Village, south of Zichuan District (Shandong in the Qing Dynasty). He became a famous literary figure in his youth but he never passed the imperial examinations to become an official scholar. At the age of nineteen he received the xiucai degree in the civil service examination, which entitled him to an appointment only as a government official in a township or city. It was not until he was 71 that he received the gongsheng degree. He never became wealthy in his life and he made a living as a private tutor.

To the east of Pujia Village is the Manjin Well, where, according to the legend, Pu Songling offered tea to passers-by in order to collect

Juan S. Gómez-Jeria, Lic. Q., is Associate Professor in the Faculty of Sciences at the University of Chile. This research was supported by the Department of Chemistry, Faculty of Sciences, University of Chile. Reprint requests should be addressed to Prof. Gómez-Jeria at the Faculty of Sciences, University of Chile, Casilla 653, Santiago 21, Chile; e-mail: facien03@uchile.cl. 
strange tales of different places. Later he compiled the tales and wrote them in the masterpiece called Liaozhai Zhiyi (Strange Stories from Liaozhai's Studio). It contains $\mathbf{4 7 4}$ histories of various types. There are several versions in the English language (Giles, 1880; Hsien-yi and Yang, 1981; Nyren, 1995; Page, 1991; Qingnian, Ciyun and Yi, 1997; Quong, 1946; Ruoqiang, Lunzhong and Lunjun, 1988; Soulie, 1913; Wanruo, Lee and Chingwen, 1990; Yunzhing, Tifang, Liyi and Zhihong, 1982). Some of his tales have been made into films (Ching, 1987).

In a Spanish version of Liaozhai Zhiyi (Rovetta and Ramírez, 1985), I found a short story that undoubtedly contains key elements of a neardeath experience (NDE), which I now present.

\section{The Report of the Near-Death Experience}

The story is entitled "Dr. Tang." I present it below, separated into steps in their order of appearance.

(1) Tang was in bed for a long time because of an illness.

(2) Suddenly he felt an intense wave of heat rising gradually from his lower extremities.

(3) When the heat wave arrived at his hips his feet were already numb, and when the wave reached his stomach his legs were numb.

(4) When the heat arrived to his heart all the episodes of his life appeared in front of him, transported in his blood.

(5) If he remembered a commendable action he had a delicious sensation of peace; but when a reprehensible one appeared he felt grieved and stifled as if his body were a brimming cauldron of boiling oil.

(6) Once all the episodes of his life had passed in sequence in his memory, the heat went up through his throat, entered his brain, and left it through the top of his head.

(7) Several hours later his spirit abandoned his body and began to roam in the outskirts of the village.

(8) The story runs here through a series of adventures. He found a giant who put him inside his sleeve in the midst of a crowd of spirits. Tang found this situation horrible and stifling. He appealed to Buddha to get out of his situation. After the encounter with the giant, he found a bonzo who informed him 
that the Record of Life and Death was in the hands of King Wen Yang and Confucius the Wise. He then met Confucius, who sent him to Wen Yang's palace.

(9) The King said to Tang that he had the right to live many years more but that his mortal body had decomposed. He sent Tang to see Buddha to get help.

(10) Tang arrived in the presence of Buddha (Guanyin/Avalokitesvara, Rovetta and Ramírez, 1985) and exposed his situation.

(11) After a number of requests, Buddha created a body from a willow branch and clay, and then ordered an acolyte to bring the body to "where it should be."

(12) At this moment, Tang's family heard a moan from inside his coffin, opened it, and found him resurrected. He had been dead for seven days.

\section{My Interpretation of the Report}

I suggest that the above report contains three central elements of an NDE. Points (4) and (5) correspond to the life review. Points (7) to (11) contain the almost common travel through a "spiritual world" together with the pilgrimage to obtain a healthy physical body "to return to life."

It is not known if the story was related to $\mathrm{Pu}$ Songling by the original experiencer or by someone who had heard about it. Another aspect that I cannot infer is if Pu Songling or another person added their own elements to the narration to interpret it in accordance with the beliefs of their time.

It seemed that Tang was already inside the coffin when he had his experience. This meant that people around him saw that he showed no signs of being alive and therefore they put him in a coffin to follow the proper religious rituals. Moreover it is reasonable to think that Tang had the experience not long before he began to sigh inside the coffin. How long was his experience? I cannot answer this question. Was his body really decaying? In spite of the fact mentioned in point (9) above, my answer is negative, because he was alive. If not, we should have had no report at all.

Are there any possible illnesses involving a wave of heat and numbness of the lower extremities? We should consider a sickness beginning with symptoms obliging the sufferer to rest in bed for "a 
long time" and ending in a cataleptic state lasting several days. I could not find any that would produce all of Tang's symptoms, but, as an example of the type of illness, I will cite transverse myelitis. This is a neurological disorder caused by inflammation across both sides of one level or segment of the spinal cord. Affected people sometimes report abnormal sensations such as burning in the legs, sensory loss, and paraparesis, or weakness of both legs. Paraparesis often progresses to paralysis of the legs and lower trunk. I cite this example to show only that a syndrome characterized by burning in the legs and progressive paralysis from the lower extremities to the lower part of the trunk is possible. The progressive loss of sensation of the body is one process that may lead to dissociation of mind from body. But the question of whether we are dealing with one illness followed by another one cannot be ruled out. This said, I suggest that points (1) to (3) are the prelude to the beginning of a near-death experience.

The classical life review of NDEs appears well described in point (4). Note that the episodes of his life appeared "in the front of him," which probably means "as if watching a film." The transportation by the blood of the episodes deserves a comment within the frame of traditional Chinese medicine. Traditional Chinese medicine views the human body as a dynamic energy system, in which a particular kind of energy or life force, known as qi, the equivalent of pneuma in ancient Greek medicine (Unschuld, 2003), is created and transformed. There are several varieties of $q i$ but I will not enter into details (Beijing College of Traditional Chinese Medicine, Shanghai College of Traditional Chinese Medicine, Nanjing College of Traditional Chinese Medicine, and Acupuncture Institute of the Academy of Traditional Chinese Medicine, 1980). In traditional Chinese medicine, the vessels transport blood and $q i$, the latter probably transporting the life episodes.

In traditional Chinese medicine one of the three main functions of the heart is housing the mind. The heart is considered to be the main organ governing mental activities and generalizing the physiological function of the brain. Spirit, consciousness, memory, thought, and sleep are all dominated by this function of the heart (Beijing College of Traditional Chinese Medicine, Shanghai College of Traditional Chinese Medicine, Nanjing College of Traditional Chinese Medicine, and Acupuncture Institute of the Academy of Traditional Chinese Medicine, 1980). In this context it is possible to think that the heat wave is a reinterpretation, in terms of traditional Chinese medicine, of 
an ascending burning sensation starting in the lower extremities. This is also supported by the statement in point (6) that the heat wave left Tang's body through the top of his head. This place is close to the crown chakra (called Bai Hui/One Hundred Meetings or Kun Lun Mountain/The highest peak of heaven in Taoist tradition, Sahashrara/Bodhini Chakra in Tantra). Within the framework of Oriental thought, on the top of the head lies a secondary chakra called the Brahmarandhra or nirvana chakra, the crown chakra being located some distance above the top of the head. I mention this because in Indian thought, at death, the spirit leaves the body through a tiny hole at the top of the head, called the Brahmarandhra. Similarly, in Taoist esoteric systems that teach the cultivation of an immortal spirit body, this body exits through the crown center. Therefore, it is natural that an ascending heat wave should leave the body through that place.

Point (5) is very interesting because it shows a series of pleasant and unpleasant phases in the life review. The unpleasant phase of the life review is not sufficient ground to classify this NDE a distressing NDE (Atwater, 1992; Bonenfant, 2000, 2001; Greyson and Bush, 1992; Irwin and Bramwell, 1988; Ring, 1994) because it seems to involve only events stored in one of the memory subsystems (Eichenbaum, 1997; Squire, 1992; Squire and Zola, 1996). What this specific report seems to show is that Tang was recalling only those events recorded in long-term memory and rendered recallable only after they had been tagged with emotional meanings (Richter-Levin and Akirav, 2003). This leads to the idea that the near-death experiencer recalls those events associated with strong emotional feeling and not, for example, every time he washed his face or ate a meal.

Points (6) and (7) seem to indicate that a certain interval of time passed between the life review and the beginning of the culturallyshaped phase of the NDE. I choose to call points (8) to (11) the "culturally-shaped" phase of the NDE for the following reasons. In this phase near-death experiencers describe religious, philosophical, and personal aspects of their own time and geographic places (Counts, 1983; Gómez-Jeria, 1993; Kellehear, 1993, 2001; Pasricha and Stevenson, 1986; Schorer, 1985). If we consider all the content of this phase to be accurate descriptions of real places, we are in the presence of an amazing number of "worlds beyond death." On the other hand several religious systems consider their teachings to be the authentic truth and label all others as "lies." Therefore I prefer to consider this phase as a special hallucinatory process. This hallucination is built as 
a logical history, mixing actual events with elements coming from the person's beliefs and stored in long-term memory.

Why does this happen? Evolution and natural selection provide one plausible answer (Eccles, 1992; Gómez-Jeria and Madrid-Aliste, 1996; Aboitiz and Garca, 1997; Preuss, Cáceres, Oldham and Geschwind, 2004). At any given moment of consciousness I have what is called the field of consciousness (Searle, 1992). The world, as seen in my consciousness field, is a construct sustained through the neurological processes contained within the brain. Within that field people normally pay attention to some things and not to others. Right now I am paying full attention to writing this paper, but in the periphery of my field of consciousness, for example, I am also paying some attention to the phrases coming from the television. Because of primary survival needs, our field of consciousness needs to be continuously updated. If not, we shall not survive for long. This updating is carried out by using raw data coming from the senses, previous contents of the field of consciousness, data from our memory banks, and so on. I suggest that the brain imposes the presentation of a logical and coherent updated content on the field of consciousness. Therefore, it is the breakdown of the normal connections in the brain or its abnormal functioning during the NDE (Saavedra-Aguilar and Gómez-Jeria, 1989) that alters the content of the experiencer's field of consciousness. Points (8) to (11) support the above model because the narration presented in them is logical and coherent, even if its contents are unbelievable.

The logical construction of points (8) to (11) is very interesting for the following reason. The usual NDE discovery that "it was not the time" for Tang to die was not openly stated, but was found split in two parts of points (8) to (11). Tang was "informed" that a certain Record of Life and Death was in the hands of King Wen Yang. Nothing was said about the content and the function of the Record. However, when the King informed Tang that "he has the right to live many years more," we can assume that the King obtained this information from the Record of Life and Death. In this sense the function of this Record is similar to the Book in the Mapuche NDE (Gómez-Jeria, 1993), in that it contained the lifespan of the individual. But a new element appeared in the present case. Tang's mind believed that he had been inside the coffin for too long and combined that perception with knowledge of the time a corpse takes to begin to decay. The conclusion that his body would have already started to decay contradicted the 
previous information that he was to live many years more. The only logical and coherent way to avoid this would be through the fabrication of a new physical body, the task carried out by Buddha in point (11) of the report.

Point (12) was the logical ending of points (8) to (11). Tang awoke. I cannot comment on the seven days he was apparently dead because I am not aware of cataleptic states lasting so long, and I am not sure that seven days is the right time.

Nevertheless the report contained some inconsistencies. For example, there was no logical pathway from the moment Tang felt uncomfortable in midst of the throng of spirits to the moment he was informed by a bonzo about the Record of Life and Death. No reason was provided as to why he would need that Record. Finally, the appearance of a giant, a bonzo, King Wen Yang, Confucius, and Buddha was a good example of how mythical, religious, historical, and philosophical elements can be pragmatically mixed in a way that is typical of the Chinese people.

\section{References}

Aboitiz, F., and Garca, R. (1997). The evolutionary origin of the language areas in the human brain: A neuroanatomical perspective. Brain Research Reviews, 25, 381-396.

Atwater, P. M. H. (1992). Is there a hell? Surprising observations about the near-death experience [Guest editorial]. Journal of Near-Death Studies, 10, 149-160.

Beijing College of Traditional Chinese Medicine, Shanghai College of Traditional Chinese Medicine, Nanjing College of Traditional Chinese Medicine, and Acupuncture Institute of the Academy of Traditional Chinese Medicine (eds.). (1980). Essentials of Chinese acupuncture. Beijing, China: Foreign Language Press.

Bonenfant, R. J. (2000). A near-death experience followed by the visitation of an "angellike" being. Journal of Near-Death Studies, 19, 103-113.

Bonenfant, R. J. (2001). A child's encounter with the devil: An unusual near-death experience with both blissful and frightening elements. Journal of Near-Death Studies, 20, 87-100.

Buber, M. (1991). Chinese tales: Zhuangzi, sayings and parables and Chinese ghost and love stories (Page, A., trans.). Atlantic Highlands, NJ: Humanities Press International.

Ching, S-T. (director). (1987). A Chinese ghost story [Film]. Hong Kong, China: Cinema City Film Productions, Film Workshop Ltd.

Counts, D. E. (1983). Near-death and out-of-body experiences in a Melanesian society. Anabiosis: The Journal of Near-Death Studies, 3, 115-135.

Eccles, J. C. (1992). Evolution of consciousness. Proceedings of the National Academy of Sciences, 89, 7320-7324.

Eichenbaum, H. (1992). How does the brain organize memories? Science, 277, 330-332.

Giles, H. A. (1880). Strange stories from a Chinese studio. London, England: Thomas de la Rue.

Gómez-Jeria, J. S. (1993). A near-death experience among the Mapuche people. Journal of Near-Death Studies, 11, 219-222. 
Gómez-Jeria, J. S., and Madrid-Aliste, C. (1996). Evolution and the relationship between brain and mind states. Journal of Near-Death Studies, 14, 251-272.

Greyson, B., and Bush, N. E. (1992). Distressing near-death experiences. Psychiatry, 55, 95-110.

Hsien-yi, Y., and Yang, G. (1981). Selected tales of Liaozhai. Beijing, China: Chinese Literature.

Irwin, H. J., and Bramwell, B. A. (1988). The devil in heaven: A near-death experience with both positive and negative facets. Journal of Near-Death Studies, 7, 38-43.

Kellehear, A. (1993). Culture, biology, and the near-death experience: A reappraisal. Journal of Nervous Mental Disease, 181, 148-156.

Kellehear, A. (2001). An Hawaiian near-death experience. Journal of Near-Death Studies, 20, 31-35.

Nyren, E. A. (1995). The bonds of matrimony. New York, NY: Edwin Mellen Press.

Pasricha, S., and Stevenson, I. (1986). Near-death experiences in India: A preliminary report. Journal of Nervous and Mental Disease, 174, 165-170.

Preuss, T. M., Cáceres, M., Oldham, M. C., and Geschwind, D. H. (2004). Human brain evolution: Insights from microarrays. Nature Reviews Genetics, 5, 850-860.

Qingnian, Z., Ciyun, Z., and Yi, Y. (1997). Strange tales from the Liaozhai studio. Beijing, China: People's China Publishing.

Quong, R. (1946). Chinese ghost and love stories. New York, NY: Pantheon.

Richter-Levin, G., and Akirav, I. (2003). Emotional tagging of memory formation - in the search for neural mechanisms. Brain Research Reviews, 43, 247-256.

Ring, K. (1994). Solving the riddle of frightening near-death experiences: Some testable hypotheses and a perspective based on A course in miracles. Journal of Near-Death Studies, 13, 5-23.

Rovetta, L. A., and Ramírez, L. (1985). Pu Songling: Cuentos de Liao Zhai. Madrid, Spain: Alianza Editorial.

Ruoqiang, M., Lunzhong, M., and Lunjun, M. (1988). Selected translations from $P u$ Songling's strange stories of Liaozhai. Beijing, China: Foreign Language Teaching and Research Press.

Saavedra-Aguilar, J. C., and Gómez-Jeria, J. S. (1989). A neurobiological model of neardeath experiences. Journal of Near-Death Studies, 7, 205-222.

Schorer, C. E. (1985). Two Native American near-death experiences. Omega, 16, 111-113.

Searle, J. R. (1992). The rediscovery of the mind. Cambridge, MA: Massachusetts Institute of Technology Press.

Soulie, G. (1913). Strange stories from the lodge of leisure. London, England: Constable.

Squire, L. R. (1992). Memory and the hippocampus: A synthesis from findings with rats, monkeys, and humans. Psychological Review, 99, 195-231.

Squire, L. R., and Zola, S. M. (1996). Structure and function of declarative and nondeclarative memory systems. Proceedings of the National Academy of Sciences, 93, 13515-13522.

Unschuld, P. U. (2003). Huang Di nei jing su wen: Nature, knowledge, imagery in an ancient Chinese medical text. Berkeley, CA: University of California Press.

Wanruo, X., Lee, K., and Chingwen, C. (1990). Strange stories from a Chinese studio. Taipei, Taiwan: Hilit Publishing.

Yunzhing, L., Tifang, C., Liyi, Y., and Zhihong, Y. (1982). Strange tales of Liaozhai. Hong Kong, China: Commercial Press. 


\title{
MEDIA REVIEW
}

\author{
Janice M. Holden, Ed.D. \\ University of North Texas \\ Jason MacLurg, M.D. \\ Seattle, WA \\ Debbie James, M.S.N., R.N., C.C.R.N., C.N.S. \\ University of Texas M. D. Anderson Cancer Center
}

\begin{abstract}
The Day I Died: The Mind, the Brain, and Near-Death Experiences [videorecording], produced by Kate Broome. British Broadcasting Corporation, 2002; color; running time: 60 minutes. Available for purchase in the United States in DVD or VHS format for use in educational venues at: http://www.films.com/id/ 11685 (\$149.95 + \$12 shipping) or, for members of the International Association for Near-Death Studies, by calling 860-882-1211 ( $\$ 89.95+$ $\$ 12$ shipping).
\end{abstract}

In the fall of 2003, The Learning Channel premiered a British Broadcasting Corporation (BBC) program entitled The Day I Died: The Mind, the Brain, and Near-Death Experiences. Immediately following the broadcast, several members of the Board of Directors of the International Association for Near-Death Studies (IANDS) were in email communication expressing excitement about the quality of the program. The consensus was that, although not perfect, The Day I Died came the closest yet to being the long-sought "ultimate" NDE educational video. Through IANDS Board efforts, in conjunction with the BBC and Films for Humanities and Sciences (FHS), this program

Janice Holden, Ed.D., is Professor of Counseling and Interim Chair of the Department of Counseling, Development, and Higher Education in the College of Education at the University of North Texas in Denton, TX. Jason MacLurg, M.D., is a psychiatrist in private practice in Seattle, WA. Debbie James, M.S.N., R.N., C.C.R.N., C.N.S. is Critical Care Nurse and Manager of Clinical Nursing Instructors at the University of Texas M. D. Anderson Cancer Center in Houston, TX. Reprint requests should be addressed to Dr. Holden at the College of Education, University of North Texas, P. O. Box 310829, Denton, TX 76203-0829; e-mail: holden@coe.unt.edu. 
has recently become available on DVD and VHS. According to the terms of release, it is available for purchase in the United States for use in educational venues, though not for individual home viewing. The program is available at a substantial discount to qualifying purchasers who are IANDS members. In this review, three IANDS Board members - a professional counselor (JH), a critical care nurse and nursing instructor (DJ), and a psychiatrist (BJM) - explain our conclusion that The Day I Died, though produced for a popular audience, has substantial merit for a variety of venues, including academic and medical.

\section{Summary of Content}

The one-hour documentary film follows a specific outline, beginning with a brief, somewhat sensationalized introduction to the popular history of the topic, but then quickly turns to the serious. Questions raised by near-death experiencers (NDErs) and the scientists who study them threaten to transform the very foundation of Western scientific thought. Being unable to answer definitively the scientific speculation, the film shifts to what research has clearly revealed: that an NDE is a profound event that can have life-changing effects on the people whom they touch and often on those who strive to understand their meaning.

The Day I Died begins with a visual representation of movement through darkness toward a light, while a woman's voice, which we later learn is the voice of NDEr Pam Reynolds, provides a brief, poignant recollection from her NDE. Then, while a cardiac resuscitation scene is briefly reenacted, a calm and reassuring narrator tells viewers that reports of NDEs are increasingly common due to advances in resuscitation technology. Viewers meet several other experiencers with brief, interesting, and dramatic vignettes from their own NDEs.

Viewers next meet Dutch cardiologist and consciousness researcher Pim van Lommel. His landmark prospective study of more than 300 cardiac arrest survivors was published in the prestigious British medical journal The Lancet (van Lommel, van Wees, Meyers, and Elfferich, 2001). He states the premise of the program:

Till now, the concept was that the brain is the producer of consciousness and the producer of memories.... When you study near-death experience, you have to ... reconsider this concept, that 
perhaps we should consider the brain not as a producer but as a receiver of consciousness. And that's a kind of revolution.

The narrator then poses the focal question of the program: "Could the NDE be happening when the brain is not functioning, when the person is clinically dead?"

Next, the program provides a brief history of near-death studies, beginning with the seminal 1975 work of psychiatrist Raymond Moody, Life after Life. Then Bruce Greyson, psychiatrist at the University of Virginia, describes his NDE Scale (Greyson, 1983), which provided an important quantitative and qualitative standard that was needed to study scientifically the scope and depth of NDE phenomena. After his description of each of the four subscales of the instrument, brief clinical examples from actual case reports illustrate the focus of the NDE scale.

The first of four in-depth case studies is then presented, that of Heather Sloan of the United Kingdom. Her case, involving complications arising from an ectopic pregnancy, illustrates several classic features of a typical NDE.

The next focus of the program is the research of Sam Parnia, who was at that time cardiologist at Southampton Hospital in the United Kingdom, who claims that his team's prospective study was "the first ever study that was published in cardiac arrest patients." His coauthor, neuropsychiatrist Peter Fenwick, then restates the central scientific dilemma posed by the NDE: "Once the heart has stopped, the brain ceases to function. All experience must stop. If it doesn't stop for some reason, you have a problem." The results of Parnia's and van Lommel's teams' prospective studies are briefly summarized, including a compelling case of veridical perception. Veridical perception refers to reports by the NDEr of happenings that seem physically impossible for the NDEr to have perceived, but that nevertheless are corroborated through objective sources such as medical records and/or the testimony of others who were on the scene.

Next, psychologist Susan Blackmore describes the traditionally held scientific reductionistic theory that explains NDEs in terms of the physiology of the dying brain. A case study is then presented that calls into question Blackmore's theory: that of Pam Reynolds. Her case is presented through descriptions by Atlanta cardiologist Michael Sabom and Phoenix neurosurgeon Robert Spetzler, through Reynolds's own narration, and through reenactment of the surgical procedure with particular focus on the veridical aspects of Reynolds's experience. 
Reynolds' case appears to be explored in depth at this juncture of the film in response to a crucial question Blackmore raised: When, exactly, during the crisis and resuscitation process, are NDErs' seemingly veridical perceptions occurring? The answer to that question plays a critical role regarding the evidentiary value for the question of consciousness functioning apart from the brain.

Stuart Hammeroff, anesthesiologist at the University of Arizona, is interviewed regarding his theory of the relationship between consciousness and the brain - a theory that draws heavily from quantum physics. He is looking particularly at microtubules in the brain and speculates on "superpositioning" and quantum entanglement to explain retained experience during a time of physical unconsciousness.

The program then turns from theory and speculation to certainty: "One thing is clear and proven," says the calm and methodical voice of the narrator, "NDEs have extreme effects on the people who've had them, because, for them, life continued after death." Greyson returns to describe these aftereffects. Typically, experiencers become less materialistic, more helpful to others, more peace-seeking, and more global-minded. This "Scrooge-like" transformation is richly illustrated in the case study of Gordon Allen of Seattle.

The last in-depth case study is that of Vicki Noratuk, blind from birth, who describes seemingly veridical visual perception during her NDE. Parnia has the last word in the program, reminding viewers that, in the history of science and medicine, what was true yesterday is not true today. He concludes that NDEs may open a new, as yet uncharted, field of consciousness studies that may very well revolutionize the scientific view of the relationship between the brain and consciousness or the mind.

\section{Strengths of the Program}

The overarching strength of this program is the skillful way that the producers presented a complex topic. They included every important aspect of research and theory related to the premise that the mind might exist and function independent of the brain. Another important strength is that the program is balanced, presenting both "believer" and "skeptic" perspectives. The producers accomplished this feat through the inclusion of many of the foremost contemporary experts on NDEs. 
A further strength is the production quality of the program that clearly reflects BBC financial and technical resources. Through the use of a world map graphic, the viewer can follow the locations of relevant sites in the Netherlands, the United Kingdom, and various places in the United States.

Regarding the content, the many personal NDE accounts told by the actual experiencers constitute a strength of the program. Represented are a variety of adults: both men and women; individuals of all three races; persons from the United States, United Kingdom, and Netherlands; and one person with the disability of blindness. Through their diversity, these individuals convey the research finding that NDEs transcend gender, race, culture, and other personal characteristics.

Collectively, the several brief testimonials and four in-depth case studies convey much of the range of NDE phenomena. Included are leaving the body through a sensation of "popping" out; the out-of-body experience including movement through "solid" objects; the life review; entry into a light; the profound and emotional experience of unconditional love; the sense that life is purposeful; encounter with deceased loved ones and other spiritual beings who communicate telepathically; the message that one has not yet accomplished what one undertook Earthly life to accomplish; and the experience of omniscience. Also described are the various circumstances of perceived return to the physical body, including a choice to return because of a love connection with a living person, being told or forced to return against one's will, and being simply and suddenly "back" in the body.

The NDE testimonials also collectively convey many of the aftereffects of NDEs. Various disclosure patterns are represented, including nondisclosure for years after the experience, disclosure to a physician, and a compulsion to disclose the experience to numerous people. Also conveyed are a knowing conviction that conscious existence continues after physical death, resulting in a complete loss of the fear of death; a moment-to-moment ongoing sense of connection to that which is "higher"; and the relinquishing of materialistic goals in favor of altruistic ones.

The producer's choice of Heather Sloane to provide the first testimonial was masterful: Her unassuming manner, warmth, twinkling eyes, humility, groundedness, and soft-spoken earnestness make her both likable and credible. Each of the in-depth case studies added important new features to the previous ones. 
An added strength of the FHS package is a 20-page Teacher's Guide/ Viewing Guide available for free at http://www.iands.org/news/latest/ the_ultimate_video.html by clicking on "Teacher's/viewing Guide" or at http://www.films.com/id/11685\# by clicking on "related resources." The Guide includes an Introduction, Learning Objectives, Educational Standards, Program Overview, Main Topics, Fast Facts about NDEs, Vocabulary Terms, Pre- and Post-Program Discussion Questions, Group Activities, and Additional Resources. Equipped with this resource, an instructor or discussion leader could enhance the onehour of viewing time with up to an additional hour of meaningful interaction among viewers.

Perhaps one of the greatest strengths of the program is its use of eminent physicians and academicians whose presence lends credibility to the field of near-death studies. Professionals in the field, aware that research on NDEs and NDErs has been ongoing for over three decades, know that such credibility is deserved. But because NDEs involve transpersonal phenomena that challenge the fundamental scientific assumptions of Western culture and to some degree defy purely scientific inquiry, and because they include aspects that are generally recognized to be of a religious or spiritual nature, a domain that has, for so long, fallen outside the bounds of scientific inquiry, the public at large, and many professionals, may not have perceived NDEs as a valid subject of scientific pursuit. Thus, one of the great strengths of this program is its potential to legitimize NDEs as a topic for discussion, research, and application among the public, researchers, and teachers, as well as medical, mental, and spiritual healthcare providers.

\section{Limitations of the Program}

It was beyond the apparent intent of the producer to provide a thorough introduction to all NDE-related phenomena. Because they focused exclusively on pleasurable NDEs, those dominated by feelings of peace, bliss, and love, the program includes no mention of distressing NDEs, those dominated by feelings of terror, horror, or isolation. Although distressing experiences seem to constitute a small minority of all NDEs, they appear to be equally real to experiencers, and the needs of distressing NDErs in the aftermath of their experiences seem equally, if not more, pressing as those of pleasurable NDErs. Thus, as a stand-alone introduction to NDEs, this program 
falls short on this point. Fortunately, the Teacher's/Viewing Guide addresses this phenomenon, including how to find further resources. However, nothing guarantees that a user of this program will attend to that aspect of the Guide or make sure that at least a mention is made of the reality and importance of distressing NDEs.

Another limitation is a fine point of accuracy regarding the history of prospective research with cardiac arrest patients. In fact, Parnia's team's 2001 study was not, as stated in the program, the first-ever of its kind. In 1988, Janice Holden discussed the rationale and procedures for such research, specifically to study veridical perception among cardiac arrest patients in hospital; in 1990, Holden and Leroy Joesten reported on their study of this type at Lutheran General Hospital in Park Ridge, IL; and in 1996, Madelaine Lawrence reported on a similar study she conducted at Hartford Hospital in Hartford, CT. In Parnia's defense, theirs was the first study of cardiac arrest patients to capture reports of NDEs. A point of possible interest is that, like Parnia's study, other such attempts at veridical perception research have yielded inconclusive results. Because the purpose of The Day I Died was not to inform professional researchers thoroughly about how to undertake this kind of research but was, rather, to promote interest in the potential for such research, the omission of this historical information does not detract from the overall value and impact of the program.

Other limitations of the program relate specifically to potential venues in which it may be shown. Audiences more interested in the experiential phenomenon of the NDE may react negatively to the more clinical and scientific aspects of the program. Conversely, highly clinically and scientifically oriented audiences may react negatively to some of the production techniques aimed at popular audiences, such as the somewhat sensationalized introductory minute or two of the program, and the repeated use of the geography graphic throughout the program.

\section{Conclusion}

Despite its limitations, we consider this program to be an excellent introduction to NDEs, current research on them, and the questions they raise about the nature of human consciousness, for inquiring audiences, both general and professional. For an audience more interested in the experiential aspects of NDEs, a presenter might 
consider a more expanded program in which attendees first view a production such as Into the Light (Engleman, 1994) or Life After Life (Shockey, 1992). But for inquiring or more advanced audiences, these latter productions alone would not provide nearly the intellectual stimulation or currency of information that The Day I Died provides.

For more professional audiences, we consider The Day I Died to be the single best audiovisual introduction to NDEs that exists to date. In fact, it is our dream that every nurse, physician, emergency medical technician, mental health professional, chaplain, clergyperson, thanatologist, brain researcher, consciousness researcher, and general psychology instructor implement this program along the lines suggested in the Teacher's/Viewing Guide. In an ideal world where this dream had become a reality, understanding of NDEs, constructive responses to NDErs, and our understanding of the nature of consciousness and its implications for a more humane world, would be greatly advanced.

\section{References}

Engleman, E. (producer/director). (1994). Into the light [videorecording]. Venice, CA: VisionQuest Video. (Available at http://www.amazon.com/gp/product/B00006JSO9/ $002-5200818-1556069 ? \mathrm{v}=$ glance $\& n=404272$ )

Greyson, B. (1983). The near-death experience scale: Construction, reliability, and validity. Journal of Nervous and Mental Disease, 171, 369-375.

Holden, J. M. (1988). Rationale and considerations for proposed near-death research in the hospital setting. Journal of Near-Death Studies, 7, 19-31.

Holden, J. M., and Joesten, L. (1990). Near-death veridicality research in the hospital setting. Journal of Near-Death Studies, 9, 45-54.

Lawrence, M. (1996). Prospective NDE studies with AIDS and cardiac patients. Paper presented at the annual conference of the International Association for Near-Death Studies, Oakland, CA.

Moody, R. (1975). Life after life. Covington, GA: Mockingbird Books.

Parnia, S., Waller, D. G., Yeates, R., and Fenwick, P. (2001). A qualitative and quantitative study of the incidence, features and aetiology of near-death experiences in cardiac arrest survivors. Resuscitation, 48, 149-156.

Shockey, P. (producer/director). (1992). Life after life [videorecording]. Nashville, TN: Cascom International. (Available at http://www.lifeafterlife.com/dvdvideo.html)

van Lommel, P., van Wees, R., Meyers, V., and Elfferich, I. (2001). Near-death experience in survivors of cardiac arrest: A prospective study in the Netherlands. Lancet, 358, 2039-2045. 